\title{
Pro-resolving lipid mediators in vascular disease
}

\author{
Michael S. Conte, ${ }^{1}$ Tejal A. Desai, ${ }^{2}$ Bian Wu, ${ }^{1}$ Melinda Schaller, ${ }^{1}$ and Evan Werlin ${ }^{1}$ \\ 'Division of Vascular and Endovascular Surgery, Department of Surgery, and Cardiovascular Research Institute, UCSF, San Francisco, California, USA. ${ }^{2}$ Department of Bioengineering and Therapeutic Sciences, \\ UCSF, San Francisco, California, USA.
}

\begin{abstract}
Unresolved inflammation is central to the pathophysiology of commonly occurring vascular diseases such as atherosclerosis, aneurysm, and deep vein thrombosis - conditions that are responsible for considerable morbidity and mortality. Surgical or catheter-based procedures performed on affected blood vessels induce acute-on-chronic inflammatory responses. The resolution of vascular inflammation is an important driver of vessel wall remodeling and functional recovery in these clinical settings. Specialized pro-resolving lipid mediators (SPMs) derived from omega-3 polyunsaturated fatty acids orchestrate key cellular processes driving resolution and a return to homeostasis. The identification of their potent effects in classic animal models of sterile inflammation triggered interest in their vascular properties. Recent studies have demonstrated that SPMs are locally synthesized in vascular tissues, have direct effects on vascular cells and their interactions with leukocytes, and play a protective role in the injury response. Early translational work has established the potential for SPMs as vascular therapeutics, and as candidate biomarkers in vascular disease. Further investigations are needed to understand the molecular and cellular mechanisms of resolution in the vasculature, to improve tools for clinical measurement, and to better define the potential for "resolution therapeutics" in vascular patients.
\end{abstract}

\section{Resolution: clinical relevance to vascular diseases} Inflammation plays a central role in the pathogenesis of cardiovascular diseases such as atherosclerosis, aneurysm disease, and venous thrombosis. Acute events and chronic complications of these diseases are frequently driven by inflammatory exacerbations and/or unresolved injurious stimuli. This JCI Review series summarizes the current state of knowledge of the molecular drivers of resolution, their biochemical and cellular pathways, and translational implications across the spectrum of human disease. In this Review we will outline how vascular disease, and clinical vascular interventions, offer a unique opportunity to leverage the evolving pharmaco-biology of resolution.

Over the last two decades, the identification of resolution as an active process with specific mediators, receptors, and downstream signaling pathways has provided a new framework for investigating mechanisms of disease as well as candidate therapeutic targets (1-3). Distinct families of specialized pro-resolving lipid mediators (SPMs), derived from polyunsaturated fatty acids (PUFAs), were identified using agnostic molecular profiling strategies in animal models of acute, self-limited inflammation (1-4). These SPM families, including the $\mathrm{E}$ - and $\mathrm{D}$-series resolvins, protectins, maresins, and lipoxins, have been sequentially characterized by structurefunction studies, and new members continue to be added (5-8). Total organic synthesis of SPMs and assessment of their physiologic effects across a range of disease and acute injury settings have followed $(3,9)$. Classic animal models of sterile inflamma-

Conflict of interest: MSC and TAD are co-inventors on a patent (US Patent and Trademark Office no. 9,463,177) assigned to Regents of the University of California and Brigham and Women's Hospital. MSC and TAD are cofounders of VasaRx. MSC has received research support from Metagenics Inc.

Reference information: J Clin Invest. 2018;128(9):3727-3735.

https://doi.org/10.1172/JCI97947. tion, such as murine chemical peritonitis, have provided a useful starting point to elucidate SPM receptors and downstream signaling pathways, and to develop quantitative measures of resolution $(2,4,8)$. Subsequent studies have demonstrated a lipid mediator "class switch" in the biochemical transition from inflammation to resolution, wherein early proinflammatory prostaglandins and leukotrienes are replaced by SPMs in exudates. The mechanisms of this shift in eicosanoid profile involve temporal changes in the expression, localization, and activity of key cellular enzymes such as 5- and 15-lipoxygenases (LOXs) (10-12). Once available, SPMs coordinate crosstalk between leukocytes and local cell populations, promoting an M1-M2 phenotypic transition in macrophages that is central to tissue repair (13). SPMs further promote resolution by positive-feedback effects on LOX activity and SPM receptor expression in leukocytes $(11,12,14)$. Translation of these concepts into therapies for complex human diseases is the current challenge faced by investigators in this exciting, evolving field. A central hypothesis of these efforts is that a relative "resolution deficit" may contribute to disease progression or limit clinical responses to existing therapies, suggesting opportunities for monitoring and/or treatments that incorporate lipid mediators (15-17).

Atherosclerosis is characterized by chronic unresolved inflammation, leading to plaque formation, progression, and downstream clinicopathologic consequences. The role of SPMs in atherogenesis is reviewed in detail elsewhere in this Review series (18). In murine and rabbit models, SPMs decrease atheroprogression and promote plaque stability (Table 1) (19-23). Peripheral arterial disease (PAD) is a common manifestation of atherosclerosis, wherein occlusive disease of the lower-extremity arteries leads to progressive disability, limb loss, and mortality. PAD is associated with aging, smoking, diabetes, dyslipidemia, and hypertension (24-28). PAD is increasing in prevalence around the globe and is currently estimated to afflict more than 200 million 
Table 1. Summary of in vivo models demonstrating effects of SPMs in vascular injury/disease

\begin{tabular}{|c|c|c|c|c|}
\hline Model type & Species & SPMs studied & Effects seen & References \\
\hline Atherosclerosis & Mouse, rabbit & $\begin{array}{l}\text { RvE1, RvD1, RvD2, MaR1, } \\
\text { 15-epi-LXA }\end{array}$ & $\begin{array}{l}\text { Reduced atherosclerosis, enhanced } \\
\text { plaque stability }\end{array}$ & $19-23$ \\
\hline Artery ligation & Mouse & RvD2, MaR1, 15-epi-LXA & Decreased neointima & 46,86 \\
\hline Angioplasty & Rat, rabbit & RvD1, RvD2 & Decreased neointima & 45,47 \\
\hline Vein bypass & Rabbit & RvD1 & Reduced vein graft hyperplasia & 48 \\
\hline Myocardial IR & Mouse, rat & RvD1, RvE1 & $\begin{array}{l}\text { Reduced infarct size, improved } \\
\text { myocardial recovery }\end{array}$ & $72-75$ \\
\hline Cerebral IR & Mouse & $\begin{array}{c}\text { 15-Epi-LXA }{ }_{4}, \mathrm{LXA}_{4}, \mathrm{RvD1}, \\
\text { RvD2, MaR1 }\end{array}$ & Reduced inflammation, mortality & $64-69$ \\
\hline Visceral IR & Mouse & $\mathrm{LXA}_{4}, \mathrm{RvD1}-3$, protectin D1 & $\begin{array}{l}\text { Reduced platelet-PMN aggregates, } \\
\text { decreased leukocyte infiltration, } \\
\text { preservation of renal function }\end{array}$ & $70,71,118,119$ \\
\hline $\begin{array}{l}\text { Hind-limb } \\
\text { ischemia }\end{array}$ & Mouse & $\mathrm{LXA}_{4}, \mathrm{RvD2}$ & Improved skeletal muscle recovery & 62,63 \\
\hline Aneurysm & Mouse & RvD1, RvD2 & Decreased aortic diameter & 50 \\
\hline
\end{tabular}

degradation and vascular smooth muscle cell (VSMC) apoptosis. At present there is no available pharmacologic treatment to reduce aneurysm growth or rupture, and surgical repair is the only effective therapy. Several studies have examined the effects of omega-3 PUFA supplementation in animal models of aortic aneurysm, demonstrating protective effects mediated by attenuation of aortic inflammation and alterations in matrix metabolism (49-51). Recent studies demonstrate that the SPMs RvD1 and RvD2 attenuate aneurysm formation and progression in murine models, in association with a reduction in metalloproteinase activity and polarization of aortic wall macrophages toward a reparative M2 phenotype (50). Clinical relevance of individuals, a roughly $25 \%$ increase over the preceding decade (25). Patients with advanced PAD exhibit a marked chronic proinflammatory state, with elevated levels of circulating biomarkers such as C-reactive protein (CRP), IL-6, soluble adhesion molecules, and monocyte chemoattractant protein-1 (MCP-1) (29-32). In early studies, we identified that circulating levels of the SPM 15-epi-lipoxin $\mathrm{A}_{4}$ (15-epi-LXA $\mathrm{A}_{4}$ ) were significantly lower in individuals with advanced PAD and correlated inversely with disease severity (15). Recent work from other investigators demonstrates that circulating levels of the SPM resolvin D1 (RvD1) or its precursor docosahexaenoic acid (DHA) are reduced in individuals with symptomatic carotid atherosclerosis (33). Thus, an imbalance between proinflammatory and pro-resolving signals may reflect or directly contribute to the progression of atherosclerotic vascular disease in humans $(15,20)$.

Surgical interventions for advanced PAD, such as angioplasty, stent placement, and bypass surgery, superimpose acute vascular injury on this chronic inflammatory substrate. The magnitude as well as temporal and spatial distribution of the ensuing inflammatory response in the vessel wall, and its resolution, are key drivers of vessel remodeling and downstream clinical outcomes (34-38). While often technically successful, the long-term outcomes of such procedures are limited by excessive vessel scarring or "restenosis" that may affect $50 \%$ or more of patients within 2 to 3 years (39-41). Elevated levels of proinflammatory cytokines have been associated with restenosis and adverse outcomes following peripheral interventions (42-44). These observations led us to hypothesize that augmentation of resolution via SPMs may mitigate the arterial injury response and reduce restenosis. Efforts in this arena have led to the preclinical development of candidate therapeutic strategies to deliver SPMs locally to arteries and bypass grafts, described in further detail below (45-48).

Aortic aneurysm is a common and lethal vascular condition characterized by protracted inflammation of the aortic wall, elevated protease activity with progressive loss of structural integrity, and impaired resolution. Infiltration of lymphocytes and monocytes/macrophages into the aortic wall promotes matrix resolution biochemistry was also shown in the context of surgical repair of aortic aneurysm, where early postoperative changes in SPM pathways were measured (52).

Deep vein thrombosis (DVT) and its sequelae are common conditions with major public health implications (53). Resolution of the acute thrombosis is linked to downstream venous remodeling and subsequent clinical post-thrombotic syndrome, a chronic disabling condition characterized by pain, swelling, skin changes, and ulceration. We speculate that SPMs may modulate the magnitude of thrombo-inflammatory events in the vasculature and participate in their subsequent resolution. For example, maresin 1 (MaR1) has been shown to enhance hemostatic function of human platelets but suppresses their inflammatory function, suggesting that SPMs may play an important role in the resolution of thrombotic events (54). Resolvin E1 (RvE1) reduces platelet activation in response to agonists such as $\operatorname{ADP}(55,56)$, and $\operatorname{RvD} 2$ treatment reduced dermal vessel thrombosis in a burn injury model (57). Early platelet-neutrophil interactions at sites of organ injury or thrombosis lead to the biosynthesis of SPMs such as MaR1, which may then stimulate the onset of resolution (58). Resolution of DVT requires phagocytosis of clot and apoptotic cells by macrophages, an activity that is increased by SPMs such as D-series resolvins and lipoxins (59). Moreover, recent work has defined a specific temporal pattern of SPM production that occurs during the formation of thrombus, which may be important to induce subsequent phases of clot resolution (60) and vessel wall remodeling (61). Collectively these data support an important role for SPMs in modulating the inflammatory response to thrombosis, and their potential to mitigate its downstream consequences.

Ischemia/reperfusion (IR) injury is a common complication of acute ischemic events, leading to ROS-dependent tissue damage. In settings such as stroke, myocardial infarction, and visceral or limb ischemia, IR injury can exert a dominant harmful effect on clinical outcomes. End-organ IR injury also affects solid organ transplants and autogenous vascular grafts. Studies in murine models of hind-limb $(62,63)$, cerebral $(64-69)$, renal $(70,71)$, and myocardial IR (72-75) have demonstrated marked attenuation of 


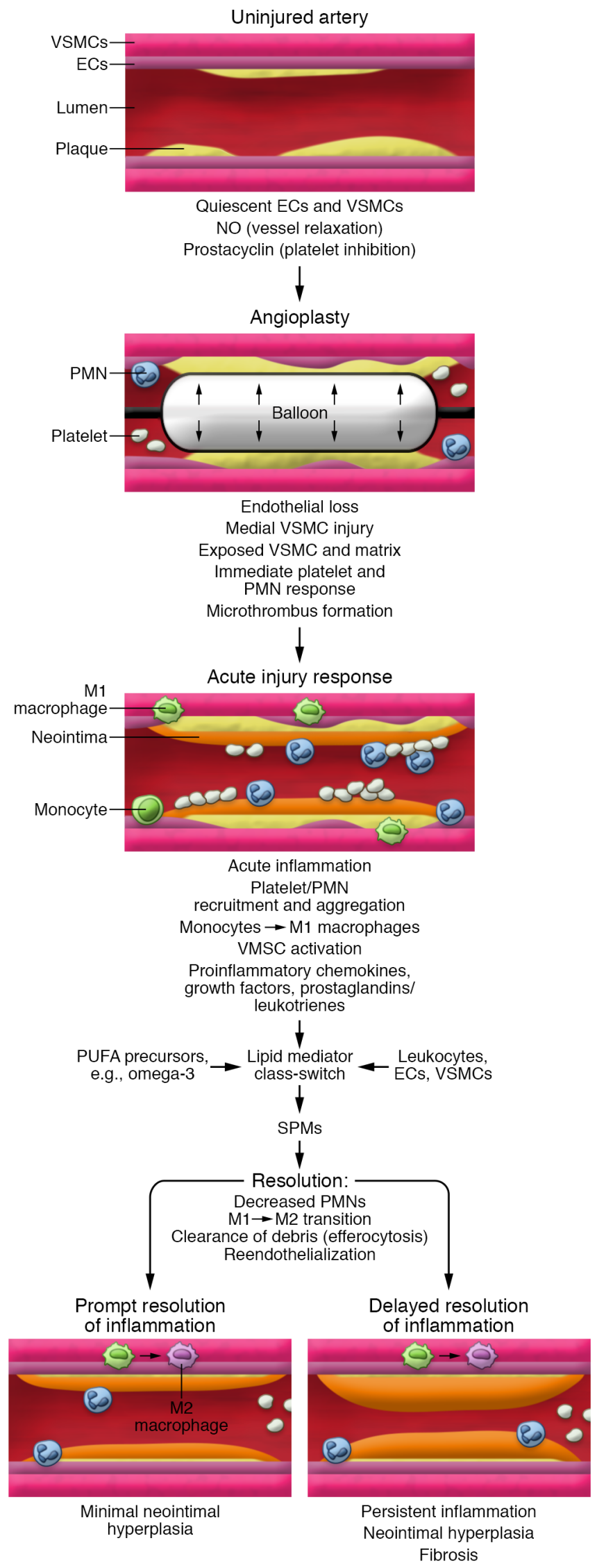

Figure 1. Resolution of vascular injury (angioplasty) and its regulation by locally derived SPM. The figure depicts injury-induced inflammation and immune response in the aftermath of arterial angioplasty. The efficiency of the resolution process that follows the acute response to vascular injury influences long-term vessel remodeling.

tissue injury by SPMs, with augmentation of cytoprotective pathways and reduced ROS signaling in a receptor-dependent manner.

\section{SPM activities and biosynthesis in the vasculature}

SPM effects on vascular cells and vascular cell-leukocyte interactions. Endothelial cells (ECs) form a continuous interface between circulating blood elements and the underlying vessel wall, regulating blood flow, platelet adhesion, and leukocyte and macromolecular egress. When ECs are disrupted or activated, a thrombo-inflammatory process ensues that is protective in settings of bacterial invasion or injury, but may have deleterious effects if recovery is impaired. Investigators have uncovered a range of effects of SPMs on ECs that collectively reduce their inflammatory activation and promote return to a quiescent state. For example, SPMs such as RvD1, RvD2, MaR1, $\mathrm{LXA}_{4}$, and protectin D1 (in picomolar to nanomolar concentrations) reduce proinflammatory cytokine production, adhesion molecule expression, and leukocyte-EC adhesion in response to a variety of inflammatory stimuli in in vitro systems $(61,76-79)$. Mechanisms identified include downregulation of NF- $\mathrm{KB}$ activation (78), induction of nitric oxide and prostacyclin synthesis (80), reduction in ROS generation (81), and involvement of the cAMP/PKA $(78,82)$ and GSK-33/C/EBP $\beta$ pathways (83). ECs express known SPM receptors including ALX/FPR2, GPR32, and GPR18 $(79,81,84,85)$. Thus the effects of SPMs on ECs may have direct relevance to chronic atherosclerosis progression and/or recovery from acute vessel injury.

VSMCs constitute the bulk of the vessel wall, providing biomechanical stability and vasomotor activity and contributing importantly to vessel wall healing. When endothelium is completely denuded (as in angioplasty injury), exposed VSMCs are also a substrate for platelet and leukocyte adhesion/recruitment. Normally quiescent VSMCs are highly organized within the arterial media, surrounded by extracellular matrix proteins. In acute vessel injury, VSMCs rapidly assume a promigratory, inflammatory, and proliferative phenotype in response to an array of locally available cytokines and growth factors, such as TNF- $\alpha$, IL-1, IL-6, platelet-derived growth factor (PDGF), fibroblast growth factor (FGF), and angiotensin II. These early events are central to the formation of neointima that leads to vessel wall thickening and subsequent reductions in lumen caliber. RvD1, RvD2, and MaR1 treatment reduces monocyte adhesion to cytokineactivated VSMCs in vitro, associated with downregulation of the expression of adhesion molecules (e.g., ICAM-1, VCAM-1) and proinflammatory genes $(45,78)$. SPMs such as $\mathrm{LXA}_{4}, \mathrm{RvD} 1, \mathrm{RvD} 2$, and RvE1 attenuate VSMC migration to a range of relevant motogens, including PDGF, thrombin, and angiotensin II $(45,47)$. These effects are receptor-dependent and involve NF- $\mathrm{kB}$ downregulation as well as activation of the cAMP/PKA pathway. We and others have identified modest antiproliferative effects of SPMs such as RvD1, RvD2, and MaR1 on VSMCs both in vitro and in vivo $(45-48,86,87)$.

SPM effects on vascular contractility. Vessel tone is continuously modulated by a balance of locally produced factors, most 

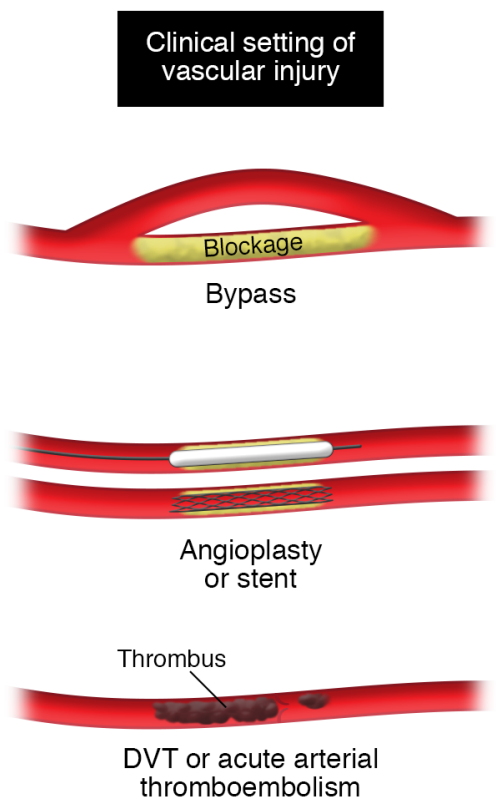
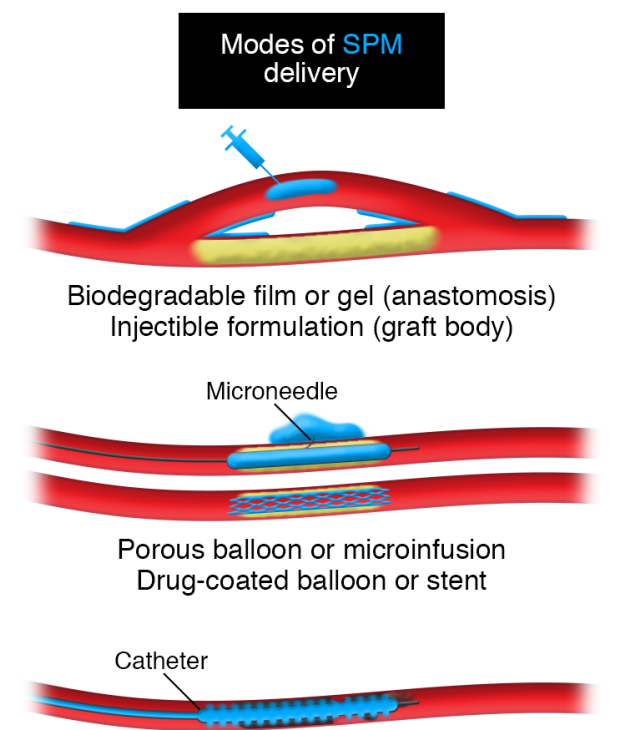

Local injectible formulation, e.g., nanoparticle SPM at time of thrombolysis/thromboembolectomy
Figure 2. Potential strategies for delivering proresolving therapeutics in clinical settings of acute vascular injury or intervention. Top: SPM-loaded films, gels, or injectable formulations can be delivered to the site of anastomosis or bypass graft. Middle: Drug-coated balloons and stents or microneedle infusions into the vascular wall can enable slow release in a site-specific manner. Bottom: Local injections via a catheter can deliver SPMs to the site of venous or arterial thrombosis concomitant with thrombolysis or thrombo-embolectomy procedures, to promote clot resolution. critically nitric oxide, which is generated by quiescent ECs and induces VSMC relaxation via soluble guanylate cyclase. In settings of injury or local inflammation, initial vasodilatory responses are important to enhance local perfusion and provide a route for neutrophils and substrates to reach target tissues. However, generation of potent vasoconstrictors in the inflammatory milieu, such as thrombin, angiotensin II, and endothelin, can promote further ischemic injury and vessel thrombosis. Recent studies have identified that certain SPMs, notably RvE1, RvD1, and RvD2, attenuate vasoconstrictive responses of human pulmonary artery and rat aortae to agonists such as thromboxane and mitigate hyperresponsiveness to extracellular calcium $(88,89)$. These responses may be important to protect against secondary ischemic insults during the phase of resolution and tissue repair. They could also be relevant to clinical settings of vasospasm that complicate surgical repair in acute traumatic injury or thromboembolic events.

Vascular injury and intimal hyperplasia. In settings of acute vessel injury such as angioplasty or bypass grafting, loss of endothelial integrity and damage to underlying VSMCs initiates a rapid response of platelets, coagulation proteins, and leukocytes, particularly neutrophils and monocytes $(34-36,38)$. These elements attach to and invade the vessel wall, elaborating an array of growth factors, proteases, cytokines, and vasoactive substances that induce secondary proinflammatory gene cascades in the surviving VSMCs and adventitial cells. Collectively these signals amplify early leukocyte recruitment and activate cellular repair programs. VSMCs undergo dramatic phenotypic transformation to an activated, migratory, and proliferative state that leads to vessel healing and neointima formation. In the early stages after injury, monocyte-derived macrophages (MDMs) in the vessel wall display an M1 phenotype, elaborating chemokines such as MCP-1 that promote further cellular recruitment. When these local inflammatory and VSMC phenotypic changes are protracted, the resultant neointimal hyperplasia is exacerbated and final lumen caliber may be reduced. In this overly simplified model (Figure 1), a resolution phase may be described during which EC regeneration occurs, VSMCs return to a quiescent state, and MDMs convert to an M2 phenotype to enhance clearance of apoptotic cells and initiate adaptive remodeling of the vessel wall. We have hypothesized that locally available SPMs are central to the resolution of vascular injury and may constitute an important homeostatic mechanism $(3,9,61,90)$.

In murine models of carotid artery ligation, neointimal hyperplasia is rapidly induced as a low-shear response in the adjacent artery. This process was markedly attenuated by systemic (i.p.) delivery of either RvD2 or MaR1, accompanied by a reduction in neutrophil and macrophage recruitment, and reduced VSMC proliferation (46). Similar observations were made with subcutaneous delivery of 15epi-LXA 4 , while no effect was seen in mice lacking the $\mathrm{LXA}_{4}$ receptor, ALX/FPR2 (86). In a rat model of carotid artery angioplasty, we found that local perivascular application of RvD1 (200 ng) using either pluronic gel or a biodegradable scaffold delivery strategy reduced neointimal hyperplasia by $50 \%$ to $60 \%$ (47). Protective effects were also demonstrated when RvD2 was delivered directly into the lumen of rabbit femoral arteries immediately after balloon angioplasty, despite highly unfavorable pharmacokinetics for this approach (45). Collectively these studies provide proof of concept for SPMs as a potential new class of candidate antirestenosis therapeutics.

Vascular biosynthesis of SPMs. Current concepts of SPM biosynthesis focus on local generation and activity as for classic autacoids. The PUFA precursors, arachidonic acid (AA), eicosapentaenoic acid (EPA), DHA, and docosapentaenoic acid (DPA), made available via local edema and in tissue exudates, are serially converted by LOXs (especially 5-LOX and 15-LOX) and cyclooxygenase (COX) pathways into bioactive lipid mediators including SPMs. These enzymatic activities may involve direct transcellular exchange of biochemical intermediates between leukocytes, platelets, and resident cells such as ECs or VSMCs. AA-derived mediators such as prostaglandins dominate the early response, and a lipid mediator class switch is hypothesized to account for the subsequent elaboration of SPMs derived from AA, EPA, DHA, and DPA $(9,91)$. These mediators act locally and may be rapidly inactivated by ubiquitous enzymes such as eicosanoid oxidoreductases and prostaglandin dehydrogenases (e.g., 15-prostaglandin dehydrogenase) (92). 


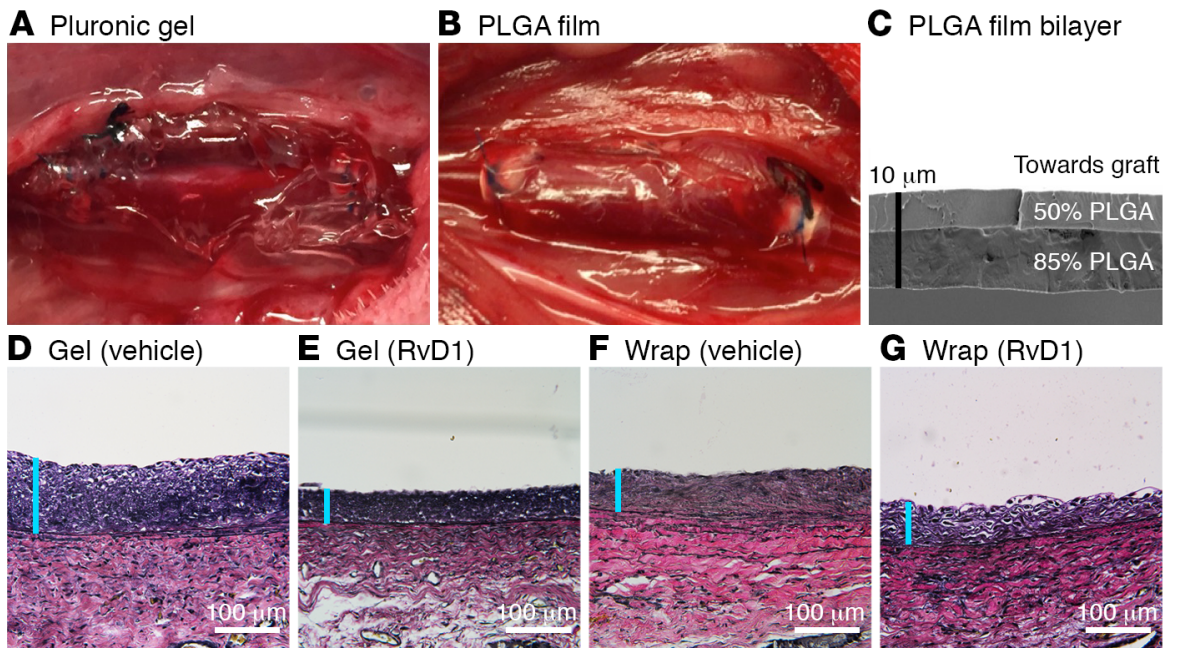

Figure 3. Perivascular delivery of RvD1 to rabbit vein grafts. (A and B) Images depict two methods of perivascular delivery of RvD1 to rabbit vein grafts (carotid interposition) using either a pluronic gel (A) or a PLGA film device (B). (C) PLGA films ("wraps") were constructed using a thin bilayered design and loaded with $1 \mu \mathrm{g}$ of RvD1; representative scanning electron microscopy of a film is shown. (D-C) Perivascular delivery of RvD1 via gel or wrap attenuated vein graft hyperplasia 28 days after implantation, whereas perivascular application of vehicle-loaded gels and wraps had no effect (elastin-stained sections). Adapted with permission from ref. 48 .
Specific sources of SPM synthesis within the vasculature remain incompletely defined. Brezinski et al. identified intraluminal LXA following coronary angioplasty in humans (93). Using liquid chromatography-tandem mass spectrometry analysis, we identified RvD1, RvD5, MaR1, and $\mathrm{LXB}_{4}$ in rabbit femoral arteries with a trend of increased SPM production early after balloon angioplasty (45). SPMs have been identified in atherosclerotic lesions of mice $(20,94)$. In recent work, we demonstrated that isolated, ex vivo human artery segments and primary cultured human vascular cells generated $\mathrm{D}$-series resolvins and maresins when the relevant fatty acid precursors (e.g., DHA, 17-hydroxydocosahexaenoic acid [17-HDHA]) were made available, and in the absence of leukocytes (95). Furthermore, conditioned media from DHA-supplemented vascular cells blunted leukocyte adhesion to cytokine-activated ECs, in a receptor-dependent fashion (i.e., ALX/FPR2 and GPR32). These studies suggest that endogenous production of SPMs within the vessel wall may represent an important paracrine pathway to actively counterregulate vascular inflammation. Further studies are needed to characterize the regulation of SPM bioavailability in blood vessels, and its influencing by dietary PUFA intake and commonly used medications such as aspirin, NSAIDs, and statins.

\section{Translation: developing a platform for pro-resolving vascular therapies}

Local delivery of SPMs is a potentially attractive approach to modulate vascular injury and circumvent the challenges of systemic pharmacokinetics and biodistribution. Although atherosclerosis is a systemic disease, surgical and interventional treatments are lesion- or vessel-focused. Based on the findings from smallanimal models described above, we have sought to develop a platform for pro-resolving vascular therapeutics leveraging polymer and device technologies (Figure 2). Using poly(lactic-co-glycolic acid) (PLGA) as a biodegradable scaffold, we described thin-film devices capable of directional elution of bioactive RvD1 over several weeks $(47,48,96)$. Others have described similar approaches using biomaterial scaffolds to locally deliver SPMs to subcutaneous tissues $(97,98)$ and bone (99). RvD1-releasing PLGA films were tested in rat carotid angioplasty (47) and rabbit vein bypass graft (48) models, demonstrating reduced early leukocyte recruitment, reduced cellular proliferation, and attenuation of downstream intimal hyperplasia (Figure 3). Injectable nanoparticle-based SPM formulations (100) may also be highly relevant, particularly if they can be targeted to localized regions of vascular damage or surgical/ interventional procedures.

\section{Clinical studies of PUFA supplementation in vascular disease}

Evidence linking dietary intake of omega-3 PUFAs to cardiovascular health has been the subject of many epidemiologic and cohort studies (101-103). The elucidation of SPM biosynthetic pathways provides a new potential mechanism for these effects. However, randomized clinical trials directly testing the "omega-3 hypothesis" in patients with cardiovascular disease or risk factors have yielded conflicting results (104, 105), and few studies have included subjects with PAD. Challenges in the interpretation of these trials include variability in formulations and dosing, as well as understanding the individual balance between individual omega-3 and omega-6 fatty acid intake and its relationship to proinflammatory versus pro-resolving lipid mediator production. Most of these trials have used lower dosing regimens of marine oil supplements, focused on downstream major clinical events such as myocardial infarction, stroke, and mortality.

Oral supplementation with omega-3 fatty acids or diets rich in their marine sources increase plasma and cell membrane levels of EPA and DHA $(106,107)$. However, the relationship between PUFA levels (or surrogate markers such as the omega-3 index [O3I]) and downstream SPM pathways is poorly understood. The physiologic impact of oral supplementation of omega-3 PUFAs is sensitive to a multiplicity of factors, including prior dietary intake and hereditary metabolic factors (108). The OMEGA-PAD-I Trial examined the effects of short-term omega-3 fatty acid supplementation on SPMs and their biosynthetic pathways in PAD patients (107). Among subjects randomized to receive $4.4 \mathrm{~g}$ of fish oil daily (2.6 $\mathrm{g}$ of EPA and $1.8 \mathrm{~g}$ of DHA) for 1 month, a significant increase was observed in the plasma levels of SPM pathway markers such as 5-hydroxyeicosapentaenoate (5-HEPE), 15-HEPE, 18-HEPE, and 4-HDHA. Secondary analyses illuminated a direct relation- 
ship between changes in the O3I and these SPM pathway markers (109). However, downstream bioactive SPMs were sparsely detected in plasma, and measures of endothelial function and systemic inflammation were unchanged following the 1-month intervention. Ongoing studies seek to examine these relationships using novel marine oil fractions and broad profiling of lipid mediator pathways and leukocyte and macrophage functions in similar populations of PAD patients (OMEGA-SPM studies; ClinicalTrials. gov identifier NCT02719665).

There have been few studies examining the effects of dietary marine oil intake and venous thromboembolism (VTE). In a population-based Norwegian cohort study, high fish consumption was associated with reduced risk of VTE, and this effect was strengthened by the use of fish oil supplements (110). Bonutti et al. reported on a prospective, nonrandomized study of orthopedic patients undergoing total knee arthroplasty $(n=850)$ receiving aspirin plus a pulsatile compression stocking, rivaroxaban, or a combination of aspirin and fish oil. The occurrence of DVT was significantly lower in the aspirin plus fish oil group versus aspirin plus stocking (0.33\% vs. $7 \%$; odds ratio $0.045 ; P<0.05)$, and not different from that in the rivaroxaban group (1\%) (111). Larger prospective, randomized, and double-blind studies are needed to determine whether omega-3 supplements have a beneficial impact on VTE.

These studies highlight a number of challenges in pursuit of the nutritional approach to augment resolution pathways. Formulations need to be standardized in terms of content of free fatty acids and downstream metabolites. Substrate utilization and biosynthesis pathways for omega-3 and omega- 6 PUFA-derived mediators are complex and involve competition for the available enzymes (3, 9, 112-116). Production of different mediator isomers can have varying potency or conflicting biologic effects. Aging and other risk factors (e.g., smoking) may alter the biosynthetic pathways of lipid mediators (117). In addition to the complexities of SPM biosynthesis, little is known about how SPM degradation pathways or SPM receptors are regulated in a tissue-specific fashion or how they may be altered in disease.

\section{Current limitations and future directions}

Based on the above summary of cell biology, animal models, and early-stage clinical investigations, the relevance of SPMs and their related pathways to vascular disease has become established and is growing. Further studies in arterial injury and restenosis, aneurysm disease, and venous thrombosis will provide much-needed insight into the mediators and processes that govern resolution in the vasculature. There are several key areas to address along the road to clinical translation.

Greater understanding of SPM receptor expression, selectivity, and downstream signaling in vascular tissues is needed to provide a stronger foundation for therapeutic targeting and pharmacokinetic studies. Rational selection of the optimal SPM and formulation for different clinical settings hinges on such efforts. Improvements in medicinal chemistry and scalability of organic synthesis are already taking place and will be pivotal to further development of SPM-based therapeutics. Locally delivered SPMs or synthetic SPM analogs may be an optimal approach for certain clinical settings, given available degradation pathways in the circulation. Nutritional supplementation with marine oil formulations enriched for SPM precursors and bioactive mediators holds promise, but will require rigorous clinical studies in vascular patients. To date, specialized instrumentation and analytics are needed to accurately measure bioactive SPMs and their precursors in tissue samples; efforts to develop more standard bench top (e.g., ELISA) assays will allow for cheaper, repeatable, and more broadly available measurement tools. Surrogate biomarkers of resolution, similar to biomarkers of inflammation (e.g., CRP, IL-6), need to be developed and validated in various disease settings to enhance preclinical and clinical study designs.

Despite these limitations, the basis for investigating SPMbased therapeutics in acute settings of vascular injury and inflammation is strong. With bioactivity in picomolar to nanomolar concentrations, a lack of discernible toxicity, and a broad profile of antiinflammatory and resolution-enhancing effects, SPMs appear particularly well suited for targeted vascular delivery across a range of clinical settings. Surgical and endovascular intervention, aneurysm disease, and acute ischemic/thrombotic events are prime targets for resolution therapeutics.

\section{Acknowledgments}

We acknowledge the support of the following research grants related to this work: HL119508 (National Heart, Lung, and Blood Institute [NHLBI]) and contract HHSN268201700005C (NHL$\mathrm{BI}$ ) to M.S. Conte. Research reported in this publication was also supported in part by a National Institute of Allergy and Infectious Diseases T32 training grant from the NIH under an award to UCSF (T32AI125222) to E. Werlin.

Address correspondence to: Michael S. Conte, Department of Surgery and Cardiovascular Research Institute, University of California, San Francisco, 555 Mission Bay Boulevard South, San Francisco, California 94143, USA. Phone: 415.353.4366; Email: Michael.conte2@ucsf.edu.
1. Serhan CN, et al. Resolvins: a family of bioactive products of omega-3 fatty acid transformation circuits initiated by aspirin treatment that counter proinflammation signals. J Exp Med. 2002;196(8):1025-1037.

2. Bannenberg GL, et al. Molecular circuits of resolution: formation and actions of resolvins and protectins. JImmunol. 2005;174(7):4345-4355.

3. Serhan CN. Pro-resolving lipid mediators are leads for resolution physiology. Nature. 2014;510(7503):92-101.
4. Schwab JM, Chiang N, Arita M, Serhan $\mathrm{CN}$. Resolvin E1 and protectin D1 activate inflammation-resolution programmes. Nature. 2007;447(7146):869-874.

5. Kohli P, Levy BD. Resolvins and protectins: mediating solutions to inflammation. Br J Pharmacol. 2009;158(4):960-971.

6. Dalli J, Colas RA, Serhan CN. Novel n-3 immunoresolvents: structures and actions. Sci Rep. 2013;3:1940.

7. Dalli J, Chiang N, Serhan CN. Elucidation of novel 13-series resolvins that increase with atorvastatin and clear infections. Nat Med. 2015;21(9):1071-1075.

8. Chiang N, Serhan CN. Structural elucidation and physiologic functions of specialized pro-resolving mediators and their receptors. Mol Aspects Med. 2017;58:114-129.

9. Serhan CN. Resolution phase of inflammation: novel endogenous anti-inflammatory and proresolving lipid mediators and pathways. Annu Rev Immunol. 2007;25:101-137. 
10. Reátegui E, et al. Microscale arrays for the profiling of start and stop signals coordinating human-neutrophil swarming. Nat Biomed Eng. 2017;1:0094.

11. Werz O, et al. Human macrophages differentially produce specific resolvin or leukotriene signals that depend on bacterial pathogenicity. Nat Commun. 2018;9(1):59.

12. Motwani MP, et al. Pro-resolving mediators promote resolution in a human skin model of UV-killed Escherichia coli-driven acute inflammation. JCI Insight. 2018;3(6):94463.

13. Dalli J, Serhan CN. Pro-resolving mediators in regulating and conferring macrophage function. Front Immunol. 2017;8:1400.

14. Fredman G, et al. Resolvin D1 limits 5lipoxygenase nuclear localization and leukotriene B4 synthesis by inhibiting a calciumactivated kinase pathway. Proc Natl Acad Sci U S A. 2014;111(40):14530-14535.

15. Ho KJ, et al. Aspirin-triggered lipoxin and resolvin E1 modulate vascular smooth muscle phenotype and correlate with peripheral atherosclerosis. Am J Pathol. 2010;177(4):2116-2123.

16. Dalli J, et al. Human sepsis eicosanoid and proresolving lipid mediator temporal profiles: correlations with survival and clinical outcomes. Crit Care Med. 2017;45(1):58-68.

17. Serhan CN, Chiang N, Dalli J. New pro-resolving $\mathrm{n}-3$ mediators bridge resolution of infectious inflammation to tissue regeneration. [published online ahead of print Sept 1,2017]. Mol Aspects Med. https://doi.org/10.1016/j. mam.2017.08.002.

18. Kasikara C, Doran AC, Cai B, Tabas I. The role of inflammation/resolution mediator imbalance in atherosclerosis. J Clin Invest. https://doi. org/10.1172/JCI97950.

19. Hasturk H, et al. Resolvin E1 (RvE1) attenuates atherosclerotic plaque formation in diet and inflammation-induced atherogenesis. Arterioscler Thromb Vasc Biol. 2015;35(5):1123-1133.

20. Fredman G, et al. An imbalance between specialized pro-resolving lipid mediators and pro-inflammatory leukotrienes promotes instability of atherosclerotic plaques. Nat Commun. 2016;7:12859.

21. Viola JR, et al. Resolving lipid mediators maresin 1 and resolvin D2 prevent atheroprogression in mice. Circ Res. 2016;119(9):1030-1038.

22. Petri $\mathrm{MH}$, et al. Aspirin-triggered lipoxin A4 inhibits atherosclerosis progression in apolipoprotein $\mathrm{E}^{-/-}$mice. Br JPharmacol. 2017;174(22):4043-4054.

23. Laguna-Fernandez A, et al. ERV1/ChemR23 signaling protects from atherosclerosis by modifying oxLDL uptake and phagocytosis in macrophages [published online ahead of print May 8, 2018]. Circulation. https://doi.org/10.1161/ CIRCULATIONAHA.117.032801.

24. Selvin E, Erlinger TP. Prevalence of and risk factors for peripheral arterial disease in the United States: results from the National Health and Nutrition Examination Survey, 1999-2000. Circulation. 2004;110(6):738-743.

25. Fowkes FG, et al. Comparison of global estimates of prevalence and risk factors for peripheral artery disease in 2000 and 2010: a systematic review and analysis. Lancet. 2013;382(9901):1329-1340.

26. Emdin CA, et al. Usual blood pressure, peripheral arterial disease, and vascular risk: cohort study of 4.2 million adults. BMJ. 2015;351:h4865.

27. Criqui MH, Aboyans V. Epidemiology of peripheral artery disease. Circ Res. 2015;116(9):1509-1526.

28. Gerhard-Herman MD, et al. 2016 AHA/ACC Guideline on the Management of Patients With Lower Extremity Peripheral Artery Disease: A Report of the American College of Cardiology/ American Heart Association Task Force on Clinical Practice Guidelines. Circulation. 2017;135(12):e726-e779.

29. Ridker PM, Buring JE, Shih J, Matias M, Hennekens $\mathrm{CH}$. Prospective study of $\mathrm{C}$-reactive protein and the risk of future cardiovascular events among apparently healthy women. Circulation. 1998;98(8):731-733.

30. Pradhan AD, Rifai N, Ridker PM. Soluble intercellular adhesion molecule-1, soluble vascular adhesion molecule-1, and the development of symptomatic peripheral arterial disease in men. Circulation. 2002;106(7):820-825

31. Tzoulaki I, Murray GD, Lee AJ, Rumley A, Lowe GD, Fowkes FG. Inflammatory, haemostatic, and rheological markers for incident peripheral arterial disease: Edinburgh Artery Study. Eur Heart J 2007;28(3):354-362.

32. Hiatt WR, et al. A validated biomarker panel to identify peripheral artery disease. Vasc Med. 2012;17(6):386-393.

33. Bazan HA, Lu Y, Jun B, Fang Z, Woods TC, Hong S. Circulating inflammation-resolving lipid mediators RvD1 and DHA are decreased in patients with acutely symptomatic carotid disease. Prostaglandins Leukot Essent Fatty Acids. 2017;125:43-47.

34. Tanaka $\mathrm{H}$, et al. Sustained activation of vascular cells and leukocytes in the rabbit aorta after balloon injury. Circulation. 1993;88(4 pt 1):1788-1803.

35. Kornowski R, Hong MK, Tio FO, Bramwell O, Wu $\mathrm{H}$, Leon MB. In-stent restenosis: contributions of inflammatory responses and arterial injury to neointimal hyperplasia. J Am Coll Cardiol. 1998;31(1):224-230.

36. Shah PK. Inflammation, neointimal hyperplasia, and restenosis: as the leukocytes roll, the arteries thicken. Circulation. 2003;107(17):2175-2177.

37. Muto A, Model L, Ziegler K, Eghbalieh SD, Dardik A. Mechanisms of vein graft adaptation to the arterial circulation: insights into the neointimal algorithm and management strategies. Circ J. 2010;74(8):1501-1512.

38. de Vries MR, Quax PHA. Inflammation in vein graft disease. Front Cardiovasc Med. 2018;5:3.

39. Conte MS, et al. Results of PREVENT III: a multicenter, randomized trial of edifoligide for the prevention of vein graft failure in lower extremity bypass surgery. J Vasc Surg. 2006;43(4):742-751; discussion 751.

40. Bradbury AW, et al. Bypass versus Angioplasty in Severe Ischaemia of the Leg (BASIL) trial: a survival prediction model to facilitate clinical decision making. J Vasc Surg. 2010;51(5 suppl):52S-68S.

41. Baumann F, Ozdoba C, Gröchenig E, Diehm $\mathrm{N}$. The importance of patency in patients with critical limb ischemia undergoing endovascular revascularization for infrapopliteal arterial dis- ease. Front Cardiovasc Med. 2014;1:17.

42. Schillinger $\mathrm{M}$, et al. Inflammatory response to stent implantation: differences in femoropopliteal, iliac, and carotid arteries. Radiology. 2002;224(2):529-535.

43. Owens CD, et al. Elevated C-reactive protein levels are associated with postoperative events in patients undergoing lower extremity vein bypass surgery. J Vasc Surg. 2007;45(1):2-9; discussion 9.

44. Joviliano EE, Piccinato CE, Dellalibera-Joviliano R, Moriya T, Évora PR. Inflammatory markers and restenosis in peripheral percutaneous angioplasty with intravascular stenting: current concepts. Ann Vasc Surg. 2011;25(6):846-855.

45. Miyahara T, et al. D-series resolvin attenuates vascular smooth muscle cell activation and neointimal hyperplasia following vascular injury. FASEB J. 2013;27(6):2220-2232.

46. Akagi D, Chen M, Toy R, Chatterjee A, Conte MS. Systemic delivery of proresolving lipid mediators resolvin D2 and maresin 1 attenuates intimal hyperplasia in mice. FASEB J. 2015;29(6):2504-2513.

47. Wu B, et al. Perivascular delivery of resolvin D1 inhibits neointimal hyperplasia in a rat model of arterial injury. J Vasc Surg. 2017;65(1):207-217.e3

48. Wu B, et al. Perivascular delivery of resolvin D1 inhibits neointimal hyperplasia in a rabbit vein graft model. J Vasc Surg. 2018. In press.

49. Wales KM, Kavazos K, Nataatmadja M, Brooks PR, Williams C, Russell FD. N-3 PUFAs protect against aortic inflammation and oxidative stress in angiotensin II-infused apolipoprotein $\mathrm{E}^{-/}$ mice. PLoS One. 2014;9(11):e112816.

50. Pope NH, et al. D-series resolvins inhibit murine abdominal aortic aneurysm formation and increase M2 macrophage polarization. FASEB J. 2016;30(12):4192-4201.

51. Meital LT, Sandow SL, Calder PC, Russell FD. Abdominal aortic aneurysm and omega-3 polyunsaturated fatty acids: mechanisms, animal models, and potential treatment. Prostaglandins Leukot Essent Fatty Acids. 2017;118:1-9.

52. Pillai PS, et al. Chemical mediators of inflammation and resolution in post-operative abdominal aortic aneurysm patients. Inflammation . 2012;35(1):98-113.

53. Office of the Surgeon General; National Heart, Lung, Blood Institute. The Surgeon General's Call to Action to Prevent Deep Vein Thrombosis and Pulmonary Embolism. Rockville, Maryland, USA Office of the Surgeon General; 2008.

54. Lannan KL, Spinelli SL, Blumberg N, Phipps RP. Maresin 1 induces a novel pro-resolving phenotype in human platelets. J Thromb Haemost. 2017;15(4):802-813.

55. Dona M, et al. Resolvin E1, an EPA-derived mediator in whole blood, selectively counterregulates leukocytes and platelets. Blood. 2008;112(3):848-855.

56. Fredman G, Van Dyke TE, Serhan CN. Resolvin E1 regulates adenosine diphosphate activation of human platelets. Arterioscler Thromb Vasc Biol. 2010;30(10):2005-2013.

57. Bohr S, Patel SJ, Sarin D, Irimia D, Yarmush ML, Berthiaume F. Resolvin D2 prevents secondary thrombosis and necrosis in a mouse burn wound model. Wound Repair Regen. 2013;21(1):35-43.

58. Abdulnour RE, et al. Maresin 1 biosynthesis during platelet-neutrophil interactions is 
organ-protective. Proc Natl Acad Sci U S A. 2014;111(46):16526-16531.

59. Elajami TK, Colas RA, Dalli J, Chiang N, Serhan CN, Welty FK. Specialized proresolving lipid mediators in patients with coronary artery disease and their potential for clot remodeling FASEB J. 2016;30(8):2792-2801.

60. Norris PC, Libreros S, Chiang N, Serhan CN. A cluster of immunoresolvents links coagulation to innate host defense in human blood. Sci Signal. 2017;10(490):eaan1471.

61. Sansbury BE, Spite M. Resolution of acute inflammation and the role of resolvins in immunity, thrombosis, and vascular biology. Circ Res. 2016;119(1):113-130.

62. Zhang MJ, et al. Resolvin D2 enhances postischemic revascularization while resolving inflammation. Circulation. 2016;134(9):666-680.

63. Zong H, Li X, Lin H, Hou C, Ma F. Lipoxin A4 pretreatment mitigates skeletal muscle ischemiareperfusion injury in rats. Am J Transl Res. 2017;9(3):1139-1150.

64. Smith HK, Gil CD, Oliani SM, Gavins FN. Targeting formyl peptide receptor 2 reduces leukocyteendothelial interactions in a murine model of stroke. FASEB J. 2015;29(5):2161-2171.

65. Han JQ, Liu CL, Wang ZY, Liu L, Cheng L, Fan YD. Anti-inflammatory properties of lipoxin A4 protect against diabetes mellitus complicated by focal cerebral ischemia/reperfusion injury. Neural Regen Res. 2016;11(4):636-640.

66. Vital SA, et al. Formyl-peptide receptor 2/3/ lipoxin A4 receptor regulates neutrophil-platelet aggregation and attenuates cerebral inflammation: impact for therapy in cardiovascular disease. Circulation. 2016;133(22):2169-2179.

67. Xian W, et al. The pro-resolving lipid mediator Maresin 1 protects against cerebral ischemia/ reperfusion injury by attenuating the proinflammatory response. Biochem Biophys Res Commun. 2016;472(1):175-181.

68. Bisicchia E, et al. Resolvin D1 halts remote neuroinflammation and improves functional recovery after focal brain damage Via ALX/FPR2 receptor-regulated microRNAs [published online ahead of print January 22, 2018]. Mol Neurobiol. https://doi.org/10.1007/s12035-018-0889-z.

69. Zuo G, et al. Resolvin D2 protects against cerebral ischemia/reperfusion injury in rats. Mol Brain. 2018;11(1):9.

70. Duffield JS, et al. Resolvin D series and protectin D1 mitigate acute kidney injury. J Immunol. 2006;177(9):5902-5911.

71. Jaworska K, et al. Both PD-1 ligands protect the kidney from ischemia reperfusion injury. J Immunol. 2015;194(1):325-333.

72. Keyes KT, et al. Resolvin E1 protects the rat heart against reperfusion injury. Am J Physiol Heart Circ Physiol. 2010;299(1):H153-H164.

73. Gilbert K, Bernier J, Godbout R, Rousseau G. Resolvin D1, a metabolite of omega-3 polyunsaturated fatty acid, decreases post-myocardial infarct depression. Mar Drugs. 2014;12(11):5396-5407.

74. Kain V, et al. Resolvin D1 activates the inflammation resolving response at splenic and ventricular site following myocardial infarction leading to improved ventricular function. J Mol Cell Cardiol. 2015;84:24-35.
75. Liu G, et al. Early treatment with Resolvin E1 facilitates myocardial recovery from ischaemia in mice. Br J Pharmacol. 2018;175(8):1205-1216.

76. Filep JG, Zouki C, Petasis NA, Hachicha M, Serhan CN. Anti-inflammatory actions of lipoxin A(4) stable analogs are demonstrable in human whole blood: modulation of leukocyte adhesion molecules and inhibition of neutrophil-endothelial interactions. Blood.1999;94(12):4132-4142.

77. Merched AJ, Ko K, Gotlinger KH, Serhan CN, Chan L. Atherosclerosis: evidence for impairment of resolution of vascular inflammation governed by specific lipid mediators. FASEB J. 2008;22(10):3595-3606.

78. Chatterjee A, Sharma A, Chen M, Toy R, Mottola $\mathrm{G}$, Conte MS. The pro-resolving lipid mediator maresin 1 (MaR1) attenuates inflammatory signaling pathways in vascular smooth muscle and endothelial cells. PLoS One. 2014;9(11):e113480.

79. Chattopadhyay R, Mani AM, Singh NK, Rao GN. Resolvin D1 blocks H2O2-mediated inhibitory crosstalk between SHP2 and PP2A and suppresses endothelial-monocyte interactions. Free Radic Biol Med. 2018;117:119-131.

80. Spite M, et al. Resolvin D2 is a potent regulator of leukocytes and controls microbial sepsis. Nature. 2009;461(7268):1287-1291.

81. Chattopadhyay R, Raghavan S, Rao GN. Resolvin D1 via prevention of ROS-mediated SHP2 inactivation protects endothelial adherens junction integrity and barrier function. Redox Biol. 2017;12:438-455

82. Mottola G, Chatterjee A, Wu B, Chen M, Conte MS. Aspirin-triggered resolvin D1 attenuates PDGF-induced vascular smooth muscle cell migration via the cyclic adenosine monophosphate/ protein kinase A (cAMP/PKA) pathway. PLoS One. 2017;12(3):e0174936.

83. Maekawa T, et al. Antagonistic effects of IL-17 and D-resolvins on endothelial Del-1 expression through a GSK-3 $\beta-\mathrm{C} / \mathrm{EBP} \beta$ pathway. Nat Commun. 2015;6:8272.

84. Chiang N, Arita M, Serhan CN. Anti-inflammatory circuitry: lipoxin, aspirin-triggered lipoxins and their receptor ALX. Prostaglandins Leukot Essent Fatty Acids. 2005;73(3-4):163-177.

85. Krishnamoorthy S, et al. Resolvin D1 binds human phagocytes with evidence for proresolving receptors. Proc Natl Acad Sci U S A. 2010;107(4):1660-1665.

86. Petri MH, Laguna-Fernandez A, Tseng CN, Hedin U, Perretti M, Bäck M. Aspirin-triggered 15-epi-lipoxin $\mathrm{A}_{4}$ signals through FPR2/ALX in vascular smooth muscle cells and protects against intimal hyperplasia after carotid ligation. Int J Cardiol. 2015;179:370-372.

87. Huynh TT, Iaccarino G, Davies MG, Svendsen E, Koch WJ, Hagen PO. Adenoviral-mediated inhibition of $\mathrm{G}$ beta gamma signaling limits the hyperplastic response in experimental vein grafts. Surgery. 1998;124(2):177-186.

88. Hiram R, et al. Resolvin E1 normalizes contractility, $\mathrm{Ca}^{2+}$ sensitivity and smooth muscle cell migration rate in TNF- $\alpha$ - and IL-6-pretreated human pulmonary arteries. Am J Physiol Lung Cell Mol Physiol. 2015;309(8):L776-L788.

89. Jannaway M, Torrens C, Warner JA, Sampson AP. Resolvin E1, resolvin D1 and resolvin
D2 inhibit constriction of rat thoracic aorta and human pulmonary artery induced by the thromboxane mimetic U46619. Br J Pharmacol. 2018;175(7):1100-1108.

90. Kreatsoulas C, Anand SS. Disparity in outcomes of surgical revascularization for limb salvage. Race and gender are synergistic determinants of vein graft failure and limb loss. Nguyen LL, Hevelone N, Rogers SO, Bandyk DF, Clowes AW, Moneta GL, Lipsitz S, Conte MS. Circulation. 2009; 119: 123-130. Vasc Med. 2009;14(4):397-399.

91. Serhan CN, Savill J. Resolution of inflammation: the beginning programs the end. Nat Immunol. 2005;6(12):1191-1197.

92. Sun YP, et al. Resolvin D1 and its aspirin-triggered 17R epimer. Stereochemical assignments, antiinflammatory properties, and enzymatic inactivation. JBiol Chem. 2007;282(13):9323-9334.

93. Brezinski DA, Nesto RW, Serhan CN. Angioplasty triggers intracoronary leukotrienes and lipoxin A4. Impact of aspirin therapy. Circulation. 1992;86(1):56-63.

94. Viola JR, et al. Resolving lipid mediators maresin 1 and resolvin D2 prevent atheroprogression in mice. Circ Res. 2016;119(9):1030-1038.

95. Chatterjee A, et al. Biosynthesis of proresolving lipid mediators by vascular cells and tissues. FASEB J. 2017;31(8):3393-3402.

96. Lance KD, et al. Unidirectional and sustained delivery of the proresolving lipid mediator resol vin D1 from a biodegradable thin film device. J Biomed Mater Res A. 2017;105(1):31-41.

97. Vasconcelos DP, Costa M, Amaral IF, Barbosa MA, Águas AP, Barbosa JN. Development of an immunomodulatory biomaterial: using resolvin D1 to modulate inflammation. Biomaterials. 2015;53:566-573.

98. Sok MCP, Tria MC, Olingy CE, San Emeterio $\mathrm{CL}$, Botchwey EA. Aspirin-triggered resolvin D1-modified materials promote the accumulation of pro-regenerative immune cell subsets and enhance vascular remodeling. Acta Biomater. 2017;53:109-122.

99. Vasconcelos DP, et al. Chitosan porous 3D scaffolds embedded with resolvin D1 to improve in vivo bone healing. J Biomed Mater Res A. 2018;106(6):1626-1633.

100. Norling LV, Spite M, Yang R, Flower RJ, Perretti $\mathrm{M}$, Serhan CN. Cutting edge: Humanized nanoproresolving medicines mimic inflammation-resolution and enhance wound healing. J Immunol. 2011;186(10):5543-5547.

101. He K, et al. Accumulated evidence on fish consumption and coronary heart disease mortality: a meta-analysis of cohort studies. Circulation. 2004;109(22):2705-2711.

102. Lavie CJ, Milani RV, Mehra MR, Ventura HO. Omega-3 polyunsaturated fatty acids and cardiovascular diseases. JAm Coll Cardiol. 2009;54(7):585-594.

103. Siscovick DS, et al. Omega-3 polyunsaturated fatty acid (fish oil) supplementation and the prevention of clinical cardiovascular disease: a science advisory from the American Heart Association. Circulation. 2017;135(15):e867-e884.

104. Rauch B, et al. OMEGA, a randomized, placebocontrolled trial to test the effect of highly purified 
omega-3 fatty acids on top of modern guidelineadjusted therapy after myocardial infarction. Circulation. 2010;122(21):2152-2159.

105. ORIGIN Trial Investigators, et al. n-3 Fatty acids and cardiovascular outcomes in patients with dysglycemia. N Engl JMed. 2012;367(4):309-318.

106. Wang $X$, et al. Effects of n-3 FA supplementation on the release of proresolving lipid mediators by blood mononuclear cells: the OmegAD study. JLipid Res. 2015;56(3):674-681.

107. Grenon SM, et al. Short-term, high-dose fish oil supplementation increases the production of omega-3 fatty acid-derived mediators in patients with peripheral artery disease (the OMEGA-PAD I Trial). J Am Heart Assoc. 2015;4(8):e002034.

108.von Schacky C. Omega-3 index and cardiovascular health. Nutrients. 2014;6(2):799-814.

109. Schaller MS, et al. Relationship between the omega-3 index and specialized pro-resolving lipid mediators in patients with peripheral arterial disease taking fish oil supplements. J Clin
Lipidol. 2017;11(5):1289-1295.

110. Hansen-Krone IJ, et al. High fish plus fish oil intake is associated with slightly reduced risk of venous thromboembolism: the Troms $\varnothing$ Study. J Nutr. 2014;144(6):861-867.

111. Bonutti PM, et al. Novel venous thromboembolic disease (VTED) prophylaxis for total knee arthroplasty-aspirin and fish oil. Ann Transl Med. 2017;5(suppl 3):S30.

112. Levy BD, Clish CB, Schmidt B, Gronert K, Serhan $\mathrm{CN}$. Lipid mediator class switching during acute inflammation: signals in resolution. Nat Immunol. 2001;2(7):612-619.

113. Spite M, Serhan CN. Novel lipid mediators promote resolution of acute inflammation: impact of aspirin and statins. Circ Res. 2010;107(10):1170-1184.

114. Merched AJ, Serhan CN, Chan L. Nutrigenetic disruption of inflammation-resolution homeostasis and atherogenesis. J Nutrigenet Nutrigenomics. 2011;4(1):12-24.

115. Colas RA, Shinohara M, Dalli J, Chiang N, Serhan $\mathrm{CN}$. Identification and signature profiles for pro-resolving and inflammatory lipid mediators in human tissue. Am J Physiol Cell Physiol. 2014;307(1):C39-C54.

116. Poorani R, Bhatt AN, Dwarakanath BS, Das UN. COX-2, aspirin and metabolism of arachidonic, eicosapentaenoic and docosahexaenoic acids and their physiological and clinical significance. Eur JPharmacol. 2016;785:116-132.

117. Halade GV, Kain V, Black LM, Prabhu SD, Ingle KA. Aging dysregulates D- and E-series resolvins to modulate cardiosplenic and cardiorenal network following myocardial infarction. Aging (Albany NY). 2016;8(11):2611-2634.

118. Brancaleone V, et al. A vasculo-protective circuit centered on lipoxin A4 and aspirin-triggered 15-epi-lipoxin A4 operative in murine microcirculation. Blood. 2013;122(4):608-617.

119. Kang JW, Lee SM. Resolvin D1 protects the liver from ischemia/reperfusion injury by enhancing M2 macrophage polarization and efferocytosis. Biochim Biophys Acta. 2016;1861(9 pt A):1025-1035. 